\title{
Poor sleep and adolescent obesity risk: a narrative review of potential mechanisms
}

This article was published in the following Dove Press journal:

Adolescent Health, Medicine and Therapeutics

\author{
Kara M Duraccio' \\ Kendra N Krietsch' \\ Marie L Chardon' \\ Tori R Van Dyk ${ }^{2}$ \\ Dean W Beebe ${ }^{1,3}$ \\ 'Cincinnati Children's Hospital Center, \\ Behavioral Medicine and Clinical \\ Psychology Department, Cincinnati, OH, \\ USA; ${ }^{2}$ Loma Linda University, \\ Department of Psychology, Loma Linda, \\ CA, USA; ${ }^{3}$ University of Cincinnati \\ College of Medicine, Department of \\ Pediatrics, Cincinnati, OH, USA
}

\begin{abstract}
Poor sleep is related to increased obesity risk in adolescents, though the mechanisms of this relationship are unclear. This paper presents a conceptual framework of the various pathways that have been proposed to drive this relationship. In this framework, increased food reward, emotional reactivity, decreased inhibitory control, metabolic disturbances, poorer dietary quality, and disrupted meal timings may increase the likelihood of increasing overall energy intake. This paper further notes how poor sleep increases sedentary behavior and screen time, which likely limits overall energy expenditure. The model posits that these mechanisms result in an imbalance of energy intake and expenditure following poor sleep, intensifying the overall risk for obesity. Increases in food reward processes, decreases in insulin sensitivity, disrupted meal timing, and increases in sedentary behavior seem to be the most compelling mechanisms linking poor sleep with increased obesity risk in adolescents. Future directions and clinical implications of this framework are discussed.
\end{abstract}

Keywords: sleep, obesity, food reward, inhibitory control, sedentary behavior, diet

\section{Introduction}

Over two-thirds of adolescents are sleep deprived. ${ }^{1}$ Sleep timing factors (eg, circadian phase delay), social factors (eg, bedtime autonomy, nighttime screen usage), and academic factors (eg, early school start times, academic pressure) place adolescents at increased risk of falling asleep late and waking up early. ${ }^{2,3}$ Short or disrupted sleep has repeatedly been linked to increased risk of developing obesity in adolescence, ${ }^{4,5}$ though the mechanisms underlying these links remain unclear.

Previous reviews of the mechanisms driving the relationship between poor sleep (defined as too little, poor quality, poorly timed, or inconsistent sleep) and obesity in youth ${ }^{6-12}$ have reflected highly variable research findings. ${ }^{6}$ Differences in child age across studies likely contribute to this variability. Adolescents are different from younger children in their cognitive abilities, ${ }^{13}$ brain development, ${ }^{14}$ desire for autonomy relating to health decision making, ${ }^{2}$ social context for eating, ${ }^{15}$ circadian phase timing and accumulation of homeostatic sleep drive, ${ }^{3}$ and overall prevalence of chronic sleep deprivation. ${ }^{3}$ Further, risk for developing obesity is high during adolescence, ${ }^{16}$ and adolescent weight status is highly predictive of adult weight status. ${ }^{17,18}$ This suggests that adolescence is a particularly important period to understand from a weight management perspective.

To our knowledge, no recent research has proposed a mechanistic model to explain the relationship between sleep and obesity in an exclusively adolescent population. Such a mechanistic model could identify novel intervention targets for
Correspondence: Kara M Duraccio Cincinnati Children's Hospital Center Behavioral Medicine and Clinical Psychology Department, 3333 Burnet Avenue, MLC 3015, Cincinnati, OH 45229-3026, USA

Tel + I 5138031387

Email Kara.Duraccio@cchmc.orgT 
adolescents and areas where future research is needed. This paper presents recent research within a multisystemic conceptual framework to highlight the pathways that may connect poor sleep to increased obesity risk in adolescents. As illustrated in Figure 1, the model includes biological, cognitive, emotional, and behavioral mechanisms that impact the balance of energy intake and expenditure, which in turn affect weight regulation. This paper first reviews how poor sleep could impact energy intake, including increased food reward, decreased inhibitory control, metabolic disturbances, and emotional reactivity, all of which may influence food selection and portion control. Next, we outline how poor sleep could limit energy expenditure via increased sedentary behavior and decreased physical activity. We posit that, in combination, these various mechanisms contribute to an energy imbalance, subsequently intensifying the overall risk for obesity.

\section{Proposed mechanisms impacting energy intake Food reward processes and hunger}

Food reward processes, or how rewarding an individual perceives or experiences food, may be impacted by poor sleep. On the neurological level, this would be reflected in sleep-related changes in the activity of reward centers of the brain. For example, studies using monetary rewards have shown alterations in reward-related neural activation in adolescents with poor sleep quality ${ }^{19}$ and variability in their sleep schedules ${ }^{20}$ though the nature of these alterations has varied. More recently, Jensen et $\mathrm{al}^{21}$ examined reward-related neural activation following experimentally shortened sleep and healthy sleep using a task involving food images in samples of normal weight and overweight adolescents. Regardless of weight class, adolescents had amplified activation in brain regions associated with food reward when experiencing shortened sleep, compared to when undergoing healthy sleep. ${ }^{21}$

Other, more phenomenological aspects of food reward include food appeal (ie, how appetizing a food appears) and food desire (ie, how strongly one desires to consume the food). ${ }^{22}$ Regarding food appeal, healthy adolescents rated sweets/desserts as more appetizing after experimentally shortened sleep than after healthy sleep. ${ }^{23}$ Another study found that weight status may affect how sleep interacts with appetitive cues; specifically, adolescents of normal weight increased their appetite ratings during experimental sleep restriction compared to healthy sleep, whereas adolescents who were overweight or obese showed high appetite ratings regardless of sleep condition. ${ }^{21}$ An intervention trial targeted to shift adolescents with habitually late bedtimes to a healthier sleep schedule also resulted in decreases in food desire for high glycemic index foods. ${ }^{24}$

Hunger is a similar construct to food reward but is more related to the physiological sensations (often discomfort or feelings of weakness) that trigger the desire to consume food. Studies evaluating relationships between sleep duration and adolescents' self-reported hunger ratings have been mixed. Experimentally shortened sleep has been associated with lower selfreported motivation to eat in adolescent boys, ${ }^{25}$ though another experimental study including both male and female youth found no change in hunger ratings based on sleep duration. ${ }^{23}$

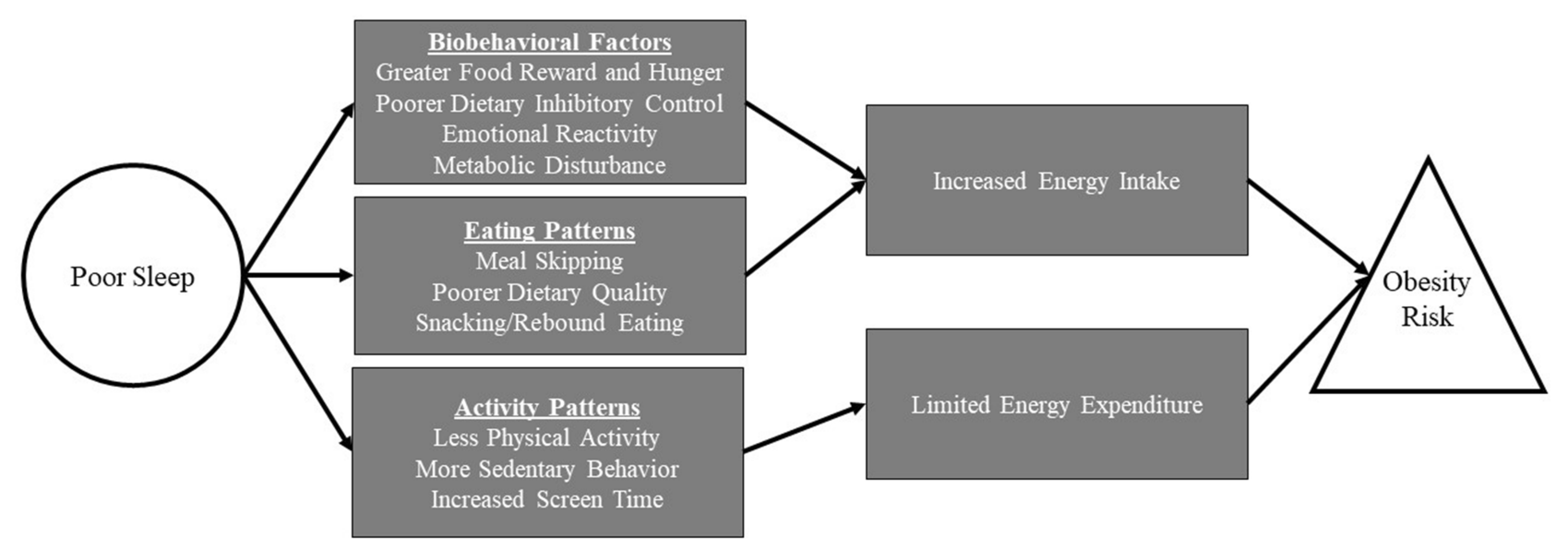

Figure I Potential mechanisms driving the relationship between poor sleep and increased obesity risk in adolescence. 
In sum, poor sleep in adolescents appears to increase neural activation in brain regions associated with food reward, appeal, and desirability, but may not increase adolescent's subjective sensation of hunger or motivation to seek out these foods. Furthermore, preliminary evidence suggests that some aspects of food reward may be improved following interventions tailored to improve sleep. Even so, all of these studies have been conducted within the past decade. Given the limited research in this area, replication is needed to draw more confident and nuanced conclusions. Most studies have used a limited range of types of foods (eg, sweets/desserts compared to fruits and vegetables) or fail to differentiate types of foods at all, so we suggest specifying additional food categories (eg, fast food, meats, snack foods) to better understand food-specific reward responses relating to sleep outcomes. Finally, recent research in the adult literature has begun to examine the relative reinforcing value of food (ie, how and when a food item is reinforcing) as an additional facet of food reward; ${ }^{26,27}$ similar methods have not yet been published in the adolescent sleep research literature.

\section{Sleep and inhibitory control}

Whereas reward processes increase appetitive drive, inhibitory control (ie, one's ability to withhold an automatic response in order to meet one's goals) can reduce dietary intake. Adolescents in developed countries often have easy access to appealing, high-calorie foods. If inadequate sleep results in diminished ability to inhibit eating such foods, this could lead to weight gain over time. Indeed, inadequate sleep has been correlated with a range of adolescent risk-taking behaviors, ${ }^{28}$ suggesting broadly decreased inhibitory control. However, findings from the few experimental studies of the impact of poor sleep on inhibitory control have been mixed. ${ }^{29}$ For example, despite having a marked effect on mood and attention, experimentally restricting healthy adolescents' sleep has been found to have minimal effect on parent- or self-reported impulsive behaviors. ${ }^{30,31}$ Amongst adolescents with attention-deficit/hyperactivity disorder, experimentally shortened sleep was found to result in greater hyperactivity/impulsivity by adolescent self-report but not by parent-report or on a computerized test. ${ }^{32}$ Computerized testing has also indicated no significant effect of a sleep treatment on response inhibition for adolescents with insomnia, despite significant benefits for attention. ${ }^{33}$

Even so, it remains possible that broad measures of inhibitory control are insensitive to specific effects of sleep on dietary inhibition or one's ability to refrain from eating a highly palatable food. Duraccio et $\mathrm{al}^{21}$ found that adolescents were less effective at discriminating healthy from unhealthy foods in a computerized inhibitory control task when they were sleep-restricted compared to when wellrested. While a direct mediation model has not been tested in adolescents, in adults, the association between weight and self-reported sleep quality is statistically mediated by self-reported dietary disinhibition. ${ }^{34}$ Furthermore, experimental sleep deprivation diminishes inhibitory control on a food-related computerized task in adults. ${ }^{35}$

Interestingly, functional neuroimaging studies have found that sleep restriction increases activation of inhibition-related cortical regions in response to food cues both in young adults ${ }^{36}$ and in normal-weight adolescents. ${ }^{37} \mathrm{On}$ the surface, such findings might suggest paradoxically better food-related inhibitory control during sleep restriction. However, Demos et $\mathrm{al}^{36}$ caution that such activation patterns reflect increased attempts at inhibiting hedonic/ reward drive but do not address whether such attempts translate to actual inhibition in day-to-day eating. Further, some individuals may not have such a compensatory attempt at inhibition; Jensen et al found that, unlike their normal-weight counterparts, adolescents who were overweight failed to show increased recruitment of inhibitory brain regions during sleep restriction. ${ }^{37}$

Thus, while the role of sleep in broadly defined behavioral inhibition remains unclear, there is at least preliminary evidence that it has more salient effects on dietary inhibition. Even so, much of that evidence is based on studies of young adults (not adolescents) that used correlational methods and indirect outcome measures (eg, food proxies such as pictures presented on a computer screen). Although generally in line with the hypothesis that inadequate sleep diminishes adolescents' ability to inhibit consumption in an obesogenic environment, more research is needed on relevant samples using real-world outcome measures.

\section{Emotional reactivity}

Emotional eating, or eating in response to negative affect, may also increase dietary intake in adolescents with poor or short sleep. Short sleep has consistently been found to worsen affective functioning in adolescents in experimental studies. ${ }^{31,38,39}$ This is consistent with neuroimaging studies in adults which have found increased amygdala activation and limbic activity in response to negative stimuli following sleep deprivation. ${ }^{40}$ Beyond worsened 
mood, shortened sleep worsens adolescent's ability to modulate emotion swings. ${ }^{31}$ Studies outside of the sleep research literature have found correlations between depressive and emotional symptoms and increased preference for, and consumption of, unhealthier foods. ${ }^{41,42}$ Eating may, at least in the short term, help stabilize emotions and improve mood, particularly for foods that heavily impact the reward centers of the brain (eg, sugary, energy-dense foods). ${ }^{43}$

Considering these findings, it is possible that adolescents may consciously or unconsciously increase their food intake as a way to regulate negative emotions experienced as a result of poor sleep. However, the few studies in adolescents that have directly examined the relationship between sleep and emotional eating have produced inconsistent results. In a large sample of adolescents from Finland, shorter sleep was associated with an increased likelihood of self-reporting eating as a way to manage stress in females but not males. ${ }^{44}$ Adolescent males from Iran who were emotional eaters had worse sleep quality, greater sleep disturbance, and worse sleep-related daytime dysfunction than those with lower levels of emotional eating. ${ }^{45}$ However, in a study comparing both pre-adolescents and adolescents who had experienced child sexual abuse with those with no abuse history, there was no relationship between emotional eating and insomnia symptoms in either group. ${ }^{46}$ Constructs closely related to emotional eating have also been examined in relation to sleep in adolescents. Disordered eating attitudes and food preoccupation have been found to be related to sleep variability in adolescents ${ }^{47}$ as well as increased sleep disturbance and daytime sleepiness in youth aged 8-17. ${ }^{48}$ Finally, greater daytime sleepiness has been associated with increased odds of binge eating in adolescent girls at-risk for developing type 2 diabetes. ${ }^{49}$

Eating as a way to regulate emotions may contribute to the relationship between poor sleep and greater dietary intake, but much research is needed before definitive conclusions can be made. In particular, there is a need for experimental sleep research and more consistent measurement of emotional eating including the use of objective, behavioral tasks in addition to self-report questionnaires. It will also be important to examine the moderating effect of individual differences beyond sex, particularly focusing on adolescents from disadvantaged backgrounds who may be at increased risk for sleep problems and obesity.

\section{Metabolic disturbances}

Metabolic processes may also be disturbed in adolescents with poor sleep. The two most studied hormones associated with appetite regulation are ghrelin (ie, a peptide hormone that stimulates appetite) and leptin (ie, a hormone that suppresses hunger). ${ }^{50}$ Although adult studies have found some evidence linking short sleep with altered profiles of leptin and ghrelin, this does not appear to generalize well to the adolescent literature. Only one observational study of adolescent females has linked shorter sleep duration to higher levels of ghrelin. ${ }^{51}$ This relationship has not been found in observational studies ${ }^{10}$ nor in an experimental sleep restriction study of adolescent males. $^{25}$ Most adolescent research has also found no consistent association between sleep and leptin levels. ${ }^{25,52,53}$ However, there may be a sex-dependent effect as one study found shorter sleep duration was associated with decreased leptin levels in adolescent boys but not girls. ${ }^{52}$

Another pathway by which sleep may impact weight may be insulin resistance or the process in which the cells in the human body become less responsive to insulin (thereby hampering the regulation of blood glucose). Although often thought of as a consequence of obesity (evident in roughly two-thirds of adolescents with obesity), ${ }^{54}$ insulin resistance has also been shown to contribute to increased dietary intake. ${ }^{55}$ Adolescents are at increased risk for developing insulin resistance simply by going through puberty. ${ }^{56}$ There is also consistent evidence that poor sleep further increases the risk of insulin resistance in adolescents. ${ }^{57-61}$ Cross-sectional research shows that adolescents who slept less than eight hours/night had higher insulin resistance compared to those who slept for eight or more hours/night. ${ }^{58,61}$ However, in an earlier adolescent study, sleeping less than eight hours/night was also associated with higher body mass index (BMI) and central distribution of fat, two markers found to attenuate the relationship between sleep and insulin sensitivity. ${ }^{62}$ Another study of adolescents with overweight/obesity found that earlier weekday bedtimes and less circadian misalignment were positively related to decreased insulin resistance, suggesting an independent effect of sleep. ${ }^{61}$ Longitudinal research also demonstrates an effect of prepubertal sleep duration and sleep efficiency on insulin sensitivity in females. ${ }^{59}$ Experimentally induced sleep restriction also increased fasting insulin levels and insulin resistance in normal-weight adolescent boys. ${ }^{60}$ 
In sum, adolescent sleep does not appear to be consistently linked with leptin and ghrelin levels. It does seem likely to impact insulin sensitivity, but no publications have tested the mediating role of insulin resistance on the relationship between poor sleep and obesity. Additional areas to explore include the potential moderating impact of sex, given the wide variability of leptin and ghrelin alterations across sexes. Importantly, future studies should be careful to control for potential nuisance or confounding variables (eg, time-of-day of blood draws, BMI, or adiposity) as hormone levels are highly sensitive to circadian effects and other difficult to control influences. See Hagan et $\mathrm{al}^{63}$ for a thorough discussion of these concerns and future directions.

\section{Measured caloric intake and eating- related behaviors \\ Caloric consumption and snacking}

Whereas there is some evidence for sleep-related differences in processes affecting caloric intake in teens, the literature linking sleep with actual daily caloric intake is not as straightforward. Cross-sectional findings are most variable, reflecting both positive and negative correlations between adolescents sleep length and caloric consumption. ${ }^{47,49,64}$ Experimental research has demonstrated that restricted sleep resulted in increases in caloric consumption in freeliving conditions ${ }^{23,65}$ but not in a highly controlled laboratory setting. ${ }^{25}$ Encouragingly, the proportion of calories consumed from foods with a low glycemic index (which induce only modest swings in blood sugar) increased following an intervention targeted to improve sleep in adolescents. ${ }^{24}$

Findings may differ depending on each adolescent's sleep phase preference (ie, "night owl" versus "morning lark"). Beebe et $\mathrm{al}^{66}$ observed that sleep phase preference moderated the relationship of experimentally manipulated sleep duration (ie, $6.5 \mathrm{hrs}$ in bed vs $9.5 \mathrm{hrs}$ in bed) and caloric intake in adolescents. Only those with an early phase preference ("morning larks") showed a reduction in caloric consumption when they got more sleep by going to bed early; those with a late phase preference ("night owls") showed no change in their caloric intake during that sleep condition. The authors also found some moderating effects of race, with White adolescents consuming more calories when sleep-restricted than non-White adolescents. While this effect did not hold after accounting for circadian preference, it did raise questions about what other environmental or contextual factors (eg, access to healthy vs unhealthy foods) may influence how the sleep/eating relationship plays out among minority adolescents. Other studies have also found that adolescents with late phase preference get more of their daily calories from snacking. ${ }^{64,67}$ These findings may suggest that circadian factors influence eating behaviors and that healthy sleep may only positively impact caloric consumption or snacking behaviors if sleep is aligned with one's internal clock.

Sleep regularity, or consistency in bedtime and wake time across weekdays and weekends, is emerging as a significant predictor of caloric consumption. He et $\mathrm{al}^{68}$ showed that greater sleep variability (but not shorter sleep duration) correlated with higher caloric consumption and intake of snacks in adolescents. That team ${ }^{69}$ also took an important next-step in the literature by showing that the relationship between sleep variability and obesity was mediated by changes in total caloric intake. Others have also linked greater sleep variability (measured by weekend oversleep) to greater caloric consumption. ${ }^{47}$ Finally, sleep quality has been shown as a unique construct that is inversely related with caloric consumption. ${ }^{70}$

In sum, discrepancies in how sleep duration affects caloric intake may be driven by several factors. We suggest that future research explores how chronotype, sleep regularity, and sleep quality impact caloric intake, as well as the nature of the research design. Clinically, before intervening at the level of sleep, it may be important to consider each individual's sleep phase preference. Promoting healthy sleep duration via earlier bedtimes may only be useful for reducing caloric intake amongst those with an early phase preference. Though snacking behavior is only beginning to be explored in this context, it is emerging as a unique eating behavior that may both be sensitive to sleep and significantly contribute to overall daily caloric intake. Future studies will need to be mindful to carefully operationalize the term snacking, as it is sometimes used to refer to eating outside of breakfast, lunch and dinner (ie, "snack bouts"), while on other occasions it is defined by the kinds of foods one eats (ie, "snack foods").

\section{Dietary quality}

Sleep has also been posited to alter the kinds of foods adolescents consume. Across multiple studies, adolescents' overall dietary quality has been found to correlate with their sleep duration, quality, and regularity. However, the definition and measurement of "dietary quality" have varied across studies. Often, researchers define dietary quality in terms of macronutrients (eg, fat, carbohydrate, 
protein) or food groupings that are more familiar to lay audiences (eg, sugar-sweetened beverages, fruits, vegetables, sweets/desserts). In general, sleep duration shows little consistent relationship to macronutrient intake in youth, with mixed and often contradictory findings across correlational studies ${ }^{6}$ and null effects in experimental work. ${ }^{65}$ Similarly, correlational data are mixed regarding sleep-related effects on consumption of fruits, vegetables, or sugar-sweetened beverages. ${ }^{71,72}$ In experimental studies, Asarnow et al reported greater consumption of fruits and vegetables and less consumption of salty food after a clinical intervention to improve adolescents' bedtimes, ${ }^{24}$ but Beebe et al reported no such effects. ${ }^{65}$

Findings have been more consistent, however, when examining sleep specifically in relation to intake of sweet/ dessert foods. Beebe et $\mathrm{al}^{23,65}$ reported that, compared to healthy sleep duration, experimentally shortened sleep nearly doubled adolescents' intake of sweet/dessert foods, but not other types of foods. Two independent research groups have found that improving sleep duration or timing decreases adolescents' consumption of high glycemic index foods (which are generally high in simple sugars, inducing rapid changes in blood glucose after consumption) - both in the free-living home environment and during an observed in-lab snack task. ${ }^{24,65}$ Similar effects have been found in several large-scale correlational studies. ${ }^{71,73-75}$

In sum, although adolescent sleep does not show a consistent relationship with macronutrient intake or certain types of foods, there is growing evidence that inadequate sleep increases consumption of sweets/desserts and other foods that are high in glycemic index. Such foods are often calorie-dense, so increased consumption has implications both for overall caloric consumption and for the development of disorders of glucose regulation. ${ }^{65}$ Given that there are documented disparities in accessibility of healthy versus highly processed foods in youth from minority or lowincome backgrounds, future work on sleep and dietary intake should also prioritize including these populations in their research. This area could also benefit from novel methods of assessing for consumption of foods at varying levels of dietary quality. For example, outcome measures could be derived from observation of behavior at food buffets to assess for real-time impacts on dietary decision making in adolescents.

\section{Meal skipping}

Beyond quantity and quality of caloric intake, meal frequency has emerged as a possible obesity risk factor in adolescents with poor sleep. De Castro et $\mathrm{al}^{76}$ proposed that by restricting an early meal (eg, breakfast), adolescents may overcompensate later in the day by eating more energy-dense foods. Short sleep duration and/or poor sleep timing might shorten morning routines, and thereby increase the likelihood of breakfast-skipping. Nearly all published studies, which include adolescents from around the world, find that shorter sleep duration, ${ }^{71,75,77-80}$ later chronotype, ${ }^{81,82}$ and poorer sleep quality ${ }^{83}$ are linked with higher rates of breakfast-skipping. Only one study found no association between breakfast skipping and sleep outcomes. ${ }^{73}$ Further, preliminary evidence suggests that shorter sleep duration may also be related to more irregular consumption of lunch and dinner ${ }^{75}$ and higher odds of eating less than three meals each day. ${ }^{84}$

Taken in context of the literature on poor sleep and food reward, meal-skipping and short sleep may interact synergistically to drive higher consumption of energydense foods at later meal occasions. Future work should attempt to investigate how sleep timing, circadian preference, meal timing, and caloric spread across meals interact at the within-person, day-to-day level. Considering the context in where adolescents eat their meals would also be beneficial. Given that nearly half of American teens qualify for free or reduced breakfast and lunch at school due to low family income, ${ }^{85}$ additional research should investigate how sleep may impact access to these meals at school.

\section{Processes impacting energy expenditure \\ Physical activity}

Potentially offsetting intake, energy expenditure (often estimated from a person's waking activity levels) represents the other side of weight homeostasis. There is intuitive reason to suspect that sleep may impact adolescents' engagement in vigorous physical activity, with downstream effects on obesity risk. However, such findings are not consistently reflected in the correlational literature. ${ }^{71,86-89}$ Several studies have found that adolescents who report sleeping $<8 \mathrm{hrs} /$ night also report engaging in fewer sports, muscle-strengthening exercises, cardiovascular activities, ${ }^{75,78,90}$ and moderate-to-vigorous physical activity than those who report sleeping $>8$ hrs. ${ }^{91}$ However, two studies found just the opposite, with longer sleep correlating with less time in objectively measured moderate-to-vigorous physical activity ${ }^{86}$ and lower levels 
of self-report high-intensity physical activity. ${ }^{71}$ Moreover, others have found no such relationship. ${ }^{87-89}$

There is notable variability in physical activity measurement across these correlational studies, making them difficult to compare. Studies with self-report measures ranged from single items (eg, "How many minutes per week do you usually spend exercising?") to well-validated questionnaires. Objective devices (eg, accelerometers) provide movement-based daily estimates of time spent in varying intensities of physical activity but do not provide context for which activities were occurring. Future studies should use complementary measures to assess physical activity from several vantage points while simultaneously allowing for greater comparability across studies. Additionally, future research may also find that physical activity is more sensitive to other sleep facets beyond sleep duration. For example, perceptions of poor sleep quality ${ }^{87}$ have predicted less engagement in physical activity. Moderating factors may also be important; Steele et $\mathrm{al}^{89}$ found that adolescents who slept in later on weekends engaged in less recreational physical activity, but only if they were also experiencing daytime sleepiness.

\section{Sedentary activity and screen time}

Though evidence related to vigorous physical activity is mixed, unhealthy sleep has been frequently linked to adolescents' sedentary behaviors, particularly while using screen-based technology. Screen time is thought to be the most "dangerous" sedentary behavior for obesity risk. ${ }^{92}$ As of nearly a decade ago, 9 out of 10 adolescents slept with at least one screen-based device (eg, phone, tablet) in their bedroom, highlighting this issue to be particularly problematic within this population relative to other age groups. ${ }^{93}$ Research has consistently pointed to a bidirectional relationship between sleep and screen time, and a 2016 metaanalysis of 20 studies found "a strong and consistent association between bedtime media use and inadequate sleep quantity, ... quality ... and excessive daytime sleepiness" across youth $6-19$ years old. ${ }^{94}$

Nearly all cross-sectional adolescent studies found that various self-reported indicators of poor sleep were predictive of more screen time. Higher levels of daytime sleepiness, ${ }^{89,95}$ later bedtime, ${ }^{74,87}$ shorter sleep duration, ${ }^{75,79,84,87,96-98}$ and greater variability in sleep ${ }^{99,100}$ have all been associated with greater self-reported total time spent watching television or playing computer or video games. Although many studies investigate the role that mobile phone usage plays on later sleep timing or problems, there is a notable dearth of research on the reverse sequence: how poor sleep might predict subsequent mobile phone use. ${ }^{101}$ This highlights one of the major challenges in this line of research; keeping current, given the speed at which technology changes.

In sum, the available literature linking sleep with screen time appears compelling but has clear limitations. Studies have been correlational and based heavily on self-report. Self-reporting may be particularly problematic as it relates to screen time use as adolescents often multi-task in their screen time use (eg, scrolling phone-based social media while doing homework on a laptop). ${ }^{102}$ This brief, unplanned, and dynamic nature of screen time may hamper adolescents' ability to estimate their use. Instead, it may be helpful to leverage electronic monitors that objectively report on daily frequency, duration, and timing of device use to get more nuanced information about potentially bidirectional relationships between sleep and screen time.

\section{Energy expenditure}

The combination of decreased physical activity and increased sedentary activity is thought to decrease overall energy expenditure, and subsequently increase obesity risk. In its purest form, energy expenditure is a measurement of the amount of energy (typically measured in kilojoules) the body burns to maintain physical functioning. Measuring energy expenditure requires highly precise, costly equipment that cannot be taken outside the lab (which limits accessibility and generalizability). To date, only one experimental study used such equipment to examine the effect of sleep on adolescents' energy expenditure. Klingenberg et $\mathrm{al}^{25}$ found that healthy adolescent boys expended more energy, had more spontaneous body movement, and consumed less daily calories compared when spending $4 \mathrm{hrs}$ in bed than when spending $9 \mathrm{hrs}$ in bed per night. While it is tempting to conclude that shortened sleep results in greater energy expenditure for adolescent boys, these results must be interpreted in the context of the highly controlled lab setting, in which adolescents had limited options for activities or dietary intake. In normal life, environment and context are paramount to adolescent behavior; when and how long adolescents sleep directly influences their daytime environment and which foods and activities they have access to. For example, an adolescent who sleeps from 1 am to 6 am on school nights may spend a greater proportion of the day awake than her peer who sleeps from $10 \mathrm{pm}$ to $6 \mathrm{am}$. She may not, however, have proportionally greater opportunities for energy-burning physical activity during the 
hours of $10 \mathrm{pm}-1 \mathrm{am}$, so there may be no change at all in such activity. Results from a small, randomized counterbalanced experimental sleep extension study may lend credibility to this theory. Van Dyk et $\mathrm{al}^{88}$ showed that increasing the total sleep duration of typically short-sleeping adolescents by over 60 mins decreased the adolescent's total daily sedentary time, but did not impact moderate or vigorous physical activity. These authors inferred that increasing sleep time does not "steal" time from opportunities to engage in energy-burning activities, but rather decreases sedentary behaviors.

\section{Strengths of proposed mechanisms}

The proposed model incorporates multisystemic mechanisms that may drive the link between poor sleep and increased obesity risk in adolescents. Overall, however, this literature is in its infancy, and future research on each is warranted. At this stage, sleep-driven increases in food reward processes, decreases in insulin sensitivity, and disrupted meal timing seem to be the most compelling mechanisms impacting energy intake. Similarly, an increase in sedentary behavior (particularly screen time) seems to be the most likely factor limiting energy expenditure after poor sleep.

Given the limitations in the existing adolescent literature (including inconsistent operationalization and measurement across constructs) and under-studied relationships, it is unclear which pathways are truly operative. Further, it remains unclear whether the multiple putative mechanisms are best viewed as part of a conditional model or a cumulative model. In a conditional model, certain conditions have to be met in order to induce increased obesity risk, whereas in a cumulative model, each mechanism could incrementally increase risk (without any single mechanism being essential). As these various pathways continue to be researched, the strength of the relationships may become clearer, and we may better understand whether conditional relationships occur.

Currently, we speculate that there are some conditional relationships within the model. For example, increases in food reward pathways might increase risk for obesity only if there is no change in, or a simultaneous decline in, inhibitory control. Jensen et $\mathrm{al}^{37}$ observed that nearly all adolescents had increases in activation in brain regions associated with food reward, but only individuals of a normal weight demonstrated increases in brain regions associated with inhibitory control. This is consistent with the idea that, unlike those with obesity, adolescents of a normal weight may experience a protective inhibitory response to counteract sleep-deprivation-induced desire or temptation. However, Jensen et al did not assess actual dietary behavior during their study. Another conditional relationship worth exploring is that of emotional reactivity and inhibitory control. While sleep restriction may result in negative emotions, it may be that adolescents who overeat in the presence of negative emotionality are also low in inhibitory control, either habitually or due to poor sleep.

\section{Additional factors for consideration}

While the current model focuses on individual-level behavioral factors that increase obesity risk, it is important to acknowledge other factors that play a large role in obesity risk and likely interact with factors in this model.

\section{Health disparities in the sleep/obesity relationship}

There is reason to suspect that racial and economic background may compound risk for sleep-related weight gain and other health disparities in adolescence. ${ }^{103}$ Research investigating sleep and weight-related behaviors has tended to focus on middle- to upper-middle class families or failed to account for socioeconomic status in analyses. However, compared to White adolescents, Black and Hispanic adolescents exhibit higher rates of obesity $(38 \%$ and $37 \%$ vs $31 \%$, respectively) ${ }^{16}$ as well as shorter sleep durations and later bedtimes. ${ }^{104}$

Although the etiology of these disparities remains uncertain, drivers are likely complex, spanning the individual, family, economic, political, and socio-cultural landscapes. ${ }^{105}$ Research has found that families from disadvantaged backgrounds may experience real, pressing, and pragmatic barriers to sleep and weight health. For example, work schedules are often outside of parental control, especially in lowerincome families, and inconsistent parent work schedules may lead to irregular use of bedtime routines and/or variability in child sleep timing. ${ }^{106,107}$ Housing insecurity or noisy, crowded, and unsafe sleeping environments also negatively impact sleep, perhaps well beyond childhood due to the development of sleep habits that adapt to the environment, but have adverse effects on health. ${ }^{106,108}$ Cultural norms and beliefs around sleep, nighttime socializing, sleep habits, and bedroom-sharing may further influence sleep practices. ${ }^{109}$ Simultaneously, low-resource neighborhoods tend to be highly "obesogenic" (ie, lacking environmental features that support healthy eating and physical activity). ${ }^{110}$ 
Low-income neighborhoods often lack accessible supermarkets with healthy food options, smaller convenience stores are more prevalent which often sell energy-dense, pre-packaged foods that are specifically marketed toward youth. As noted, there is evidence that poor sleep may decrease the ability to resist these energy-dense foods.

Overall, very little is known about how the sleep/obesity relationship works in youth from diverse backgrounds, but there is reason to suspect that this could be an important target for promoting health equity. Future research should specifically seek to recruit diverse samples and move beyond the individual to focus on the various systems that may influence both sleep and weight-related behaviors. This may include examining the built environment in neighborhoods, school start times, access to and use of free and reduced breakfast and lunch, and family and cultural beliefs about sleep and weight-related behaviors.

\section{Other potential moderating factors}

Parental obesity is one of the strongest predictors of adolescent obesity, ${ }^{111}$ likely due to increased genetic predisposition for obesity and the passing down of familial dietary practices. However, the majority of the literature presented in this review did not account for parental obesity within analyses. Further, sex is a potential moderating variable that is critical in future research. For example, in one study sleep duration predicted body mass index in adolescent males, but not adolescent females. ${ }^{112}$ Assessment of these betweensubject variables may help to elucidate moderating factors that influence the link between poor sleep and increased obesity risk.

\section{Measurement}

Most relevant research to date has been comprised of observational studies utilizing adolescent self-report of their sleep duration. Given limitations in self-report methodology and the multi-faceted nature of sleep, we recommend that future research reevaluate these associations utilizing both objective and subjective measures of sleep duration and quality. While polysomnography (PSG) is considered the gold standard for sleep assessment in the lab setting, it can be quite costly and the extensive measurement equipment and procedures could affect sleep, limiting the generalizability of the results. We suggest utilization of PSG-validated accelerometer-based assessments within future research. Additionally, given evidence of the impact of differences in sleep that extend beyond simple duration (eg, variability in sleep timing) on health factors, we suggest thoroughly testing the impact of other sleep parameters (eg, chronotype, variability in sleep timing).

\section{Clinical implications}

While the strength and the interrelations of the pathways within this model are not clear at this stage, the current state of the literature linking sleep with BMI and adiposity suggests that sleep is an appropriate target for weight loss prevention and intervention efforts within the adolescent population. In fact, previous studies have demonstrated that baseline sleep predicts the success of weight loss interventions. For example, Valrie et $\mathrm{al}^{113}$ found that adolescents with shorter baseline sleep durations and longer weekend oversleep lost less weight following a summer camp immersion treatment program than did adolescents with longer initial sleep times. Sallinen et $\mathrm{al}^{114}$ also showed that longer baseline sleep was predictive of greater weight loss following a 3-month adolescent weight loss intervention.

Sleep was not manipulated in those weight-loss trials, but Tan et $\mathrm{al}^{115}$ were able to show an effect of a sleep intervention on adolescents' body mass. By establishing and providing a set of "sleep rules" to adolescents, they were able to improve sleep hygiene, reduce daytime sleepiness, reduce overall sleep disturbances, and decrease sedentary behavior. Interestingly, they did not see impacts of their intervention on accelerometry-based measurements of sleep duration but they did see reductions in overall BMI. Other sleep-related factors (eg, sleep regularity, sleep quality, daytime sleepiness) may have played important roles. Asarnow et $\mathrm{al}^{24}$ recently conducted a pilot study to see how an intervention targeting sleep practices impacted dietary outcomes. They observed that adolescents who were able to advance their bedtimes earlier during the sleep intervention had increased consumption of low glycemic index foods (eg, fruit, dairy products) and decreased desire to consume high glycemic foods than those who were unable to advance their bedtime. However, such work remains preliminary and rigorous randomized clinical trials are needed.

Societal influences of sleep may also be intervention targets. For example, one systematic review found that by delaying early school start times by 25-60 mins, adolescents were able to add 25-77 mins (on average) of sleep per night, with additional improvements in daytime sleepiness and depression (though obesity or its mechanisms were not addressed). ${ }^{116}$ More recently, a study examining delaying school start times in Canadian children found that with every 10-min delay in start time, there was a subsequent 
decline in BMI z-score. ${ }^{117}$ Comparing high schools with start times around 8:00 am versus 9:30 am showed a difference in student BMI percentiles of the 70th versus 64th percentiles. However, it is important to keep in mind that these studies have been correlational, and there is a potential moderating impact of circadian phase preference on the impact of delayed start time. Given prior research, ${ }^{66}$ we may observe that later school start times are most beneficial for adolescents with a particularly late night preference, compared to those with earlier phase preference.

\section{Conclusions and limitations}

Poor sleep - variably defined as too little, poor quality, poorly timed, or inconsistent sleep - is related to increased obesity risk in adolescents, though the mechanisms driving this relationship are unclear. In proposing a multisystem mechanistic model specific to adolescence, we highlight several pathways by which poor sleep might increase adolescent obesity. Specifically, increased food reward and emotional reactivity, decreased inhibitory control, metabolic disturbances (eg, insulin sensitivity), and poorer dietary quality and meal timings likely increase overall energy intake. Simultaneously, we discuss how poor sleep limits vigorous physical activity and increases sedentary activity, particularly screen time, limiting overall energy expenditure.

We elected a narrative review format for this paper to allow for greater conceptual discussion than is typical of more structured systematic reviews, which are often dominated by methodological details of the review process itself and large, detailed reference tables. This choice was also motivated by the wide variety of methods employed in this relatively small and emerging research literature, which precluded a formal meta-analysis or even a less sophisticated "vote count" procedure. Even so, it is acknowledged that this review does not conform to the well-defined methodologies associated with systematic reviews (eg, PRISMA guidelines). Readers interested in a systematic review that covers similar ground but with less conceptual emphasis are directed to Krietsch et al. ${ }^{6}$ Beyond the limitations of the narrative review format, it is important to acknowledge that all reviews are dependent upon the quality of the existing empirical literature. The current paper highlights limitations in this emerging literature area, highlighting the most promising and speculative proposed mechanisms for the sleep-obesity link in adolescents. Future research needs to explore these relationships in more detail to help elucidate both the strength and the conditional relationships between these various mechanisms, as well as explore external moderators (eg, race, SES, sex, maternal obesity). These relationships can help inform current preventative and weight-loss intervention efforts in adolescent populations.

\section{Disclosure}

The authors report no conflicts of interest in this work.

\section{References}

1. Centers for Disease Control and Prevention. Short sleep duration among US adults; 2014.

2. Carskadon MA. Sleep in adolescents: the perfect storm. Pediatr Clin North Am. 2011;58(3):637-647. doi:10.1016/j.pcl.2011.03.003

3. Crowley SJ, Wolfson AR, Tarokh L, Carskadon MA. An update on adolescent sleep: new evidence informing the perfect storm model. J Adolesc. 2018;67:55-65. doi:10.1016/j.adolescence.2018.06.001

4. Miller MA, Kruisbrink M, Wallace J, Ji C, Cappuccio FP. Sleep duration and incidence of obesity in infants, children, and adolescents: a systematic review and meta-analysis of prospective studies. Sleep. 2018;41(4):zsy018. doi:10.1093/sleep/zsy024

5. Sluggett L, Wagner SL, Harris RL. Sleep duration and obesity in children and adolescents. Can J Diabetes. 2019;43(2):146-152. doi:10.1016/j.jcjd.2018.06.006

6. Krietsch KN, Chardon ML, Beebe DW, Janicke DM. Sleep and weightrelated factors in youth: a systematic review of recent studies. Sleep Med Rev. 2019;46:87-96. doi:10.1016/j.smrv.2019.04.010

7. Chen X, Beydoun MA, Wang Y. Is sleep duration associated with childhood obesity? A systematic review and meta-analysis. Obesity (Silver Spring). 2008;16(2):265-274. doi:10.1038/oby.2007.63

8. Hart CN, Cairns A, Jelalian E. Sleep and obesity in children and adolescents. Pediatr Clin North Am. 2011;58(3):715-733. doi:10.1016/j.pcl.2011.03.007

9. Lundahl A, Nelson TD. Sleep and food intake: a multisystem review of mechanisms in children and adults. $J$ Health Psychol. 2015;20(6):794-805. doi:10.1177/1359105315573427

10. Navarro-Solera M, Carrasco-Luna J, Pin-Arboledas G, GonzalezCarrascosa R, Soriano JM, Codoner-Franch P. Short sleep duration is related to emerging cardiovascular risk factors in obese children. J Pediatr Gastroenterol Nutr. 2015;61(5):571-576.

11. Felso R, Lohner S, Hollody K, Erhardt E, Molnar D. Relationship between sleep duration and childhood obesity: systematic review including the potential underlying mechanisms. Nutr Metab Cardiovasc Dis. 2017;27(9):751-761. doi:10.1016/j.numecd.2017.07.008

12. Cordova FV, Barja S, Brockmann PE. Consequences of short sleep duration on the dietary intake in children: a systematic review and metanalysis. Sleep Med Rev. 2018;42:68-84. doi:10.1016/j. smrv.2018.05.006

13. Diamond A. The early development of executive functions. In: Bialystok E, Craik FIM, editors. Lifespan Cognition. New York, NY: Oxford University Press; 2006:70-95.

14. Luna B, Padmanabhan A, O'Hearn K. What has fMRI told us about the development of cognitive control through adolescence? Brain Cogn. 2010;72(1):101-113. doi:10.1016/j.bandc.2009.08.005

15. Alberga AS, Sigal RJ, Goldfield G, Prud'homme D, Kenny GP. Overweight and obese teenagers: why is adolescence a critical period? Pediatr Obes. 2012;7(4):261-273. doi:10.1111/j.20476310.2011.00046.x 
16. Ogden CL, Carroll MD, Kit BK, Flegal KM. Prevalence of childhood and adult obesity in the united states, 2011-2012. JAMA. 2014;311(8):806-814. doi:10.1001/jama.2014.732

17. Simmonds M, Llewellyn A, Owen CG, Woolacott N. Predicting adult obesity from childhood obesity: a systematic review and meta-analysis. Obes Rev. 2016;17(2):95-107. doi:10.1111/obr.12334

18. Suchindran C, North KE, Popkin BM, Gordon-Larsen P. Association of adolescent obesity with risk of severe obesity in adulthood. Jama. 2010;304(18):2042-2047. doi:10.1001/jama.2010.1635

19. Telzer EH, Fuligni AJ, Lieberman MD, Galvan A. The effects of poor quality sleep on brain function and risk taking in adolescence. Neuroimage. 2013;71:275-283. doi:10.1016/j.neuroimage.2013.01.025

20. Hasler BP, Dahl RE, Holm SM, et al. Weekend-weekday advances in sleep timing are associated with altered reward-related brain function in healthy adolescents. Biol Psychol. 2012;91(3):334 341. doi:10.1016/j.biopsycho.2012.08.008

21. Duraccio KM, Zaugg K, Jensen CD. Effects of sleep restriction on food-related inhibitory control and reward in adolescents. J Pediatr Psychol. 2019. doi:10.1093/jpepsy/jsz008

22. Berridge KC, Ho CY, Richard JM, DiFeliceantonio AG. The tempted brain eats: pleasure and desire circuits in obesity and eating disorders. Brain Res. 2010;1350:43-64. doi:10.1016/j. brainres.2010.04.003

23. Simon SL, Field J, Miller LE, DiFrancesco M, Beebe DW. Sweet/ dessert foods are more appealing to adolescents after sleep restriction. PLoS One. 2015;10(2):e0115434. doi:10.1371/journal.pone.0115434

24. Asarnow LD, Greer SM, Walker MP, Harvey AG. The impact of sleep improvement on food choices in adolescents with late bedtimes. J Adolesc Health. 2017;60(5):570-576. doi:10.1016/j. jadohealth.2016.11.018

25. Klingenberg L, Chaput JP, Holmback U, Jennum P, Astrup A, Sjodin A. Sleep restriction is not associated with a positive energy balance in adolescent boys. Am J Clin Nutr. 2012;96(2):240-248. doi:10.3945/ajen.112.038638

26. Epstein LH, Paluch RA, Carr KA, Temple JL, Bickel WK, MacKillop J. Reinforcing value and hypothetical behavioral economic demand for food and their relation to BMI. Eat Behav. 2018;29:120-127. doi:10.1016/j.eatbeh.2018.03.008

27. Epstein LH, Dearing KK, Roba LG. A questionnaire approach to measuring the relative reinforcing efficacy of snack foods. Eat Behav. 2010;11(2):67-73. doi:10.1016/j.eatbeh.2009.09.006

28. Short MA, Weber N. Sleep duration and risk-taking in adolescents: a systematic review and meta-analysis. Sleep Med Rev. 2018;41:185-196. doi:10.1016/j.smrv.2018.03.006

29. Beebe DW. Cognitive, behavioral, and functional consequences of inadequate sleep in children and adolescents. Pediatr Clin North Am. 2011;58(3):649-665. doi:10.1016/j.pcl.2011.03.002

30. Beebe DW, Field J, Milller MM, Miller LE, LeBlond E. Impact of multinight experimentally induced short sleep on adolescent performance in a simulated classroom. Sleep. 2017;40:2. doi:10.1093/sleep/zsw035

31. Baum KT, Desai A, Field J, Miller LE, Rausch J, Beebe DW. Sleep restriction worsens mood and emotion regulation in adolescents. J Child Psychol Psychiatry. 2014;55(2):180-190. doi:10.1111/jcpp.12125

32. Becker SP, Epstein JN, Tamm L, et al. Shortened sleep duration causes sleepiness, inattention, and oppositionality in adolescents with attention-deficit/hyperactivity disorder: findings from a crossover sleep restriction/extension study. J Am Acad Child Adolesc Psychiatry. 2019;58(4):433-442. doi:10.1016/j.jaac.2018.09.439

33. de Bruin EJ, Dewald-Kaufmann JF, Oort FJ, Bogels SM, Meijer AM. Differential effects of online insomnia treatment on executive functions in adolescents. Sleep Med. 2015;16(4):510-520. doi:10.1016/j.sleep.2014.12.009

34. Blumfield ML, Bei B, Zimberg IZ, Cain SW. Dietary disinhibition mediates the relationship between poor sleep quality and body weight. Appetite. 2018;120:602-608. doi:10.1016/j.appet.2017.10.022
35. Cedernaes J, Brandell J, Ros O, et al. Increased impulsivity in response to food cues after sleep loss in healthy young men. Obesity (Silver Spring). 2014;22(8):1786-1791. doi:10.1002/oby.20786

36. Demos KE, Sweet LH, Hart CN, et al. The effects of experimental manipulation of sleep duration on neural response to food cues. Sleep. 2017;40(11):zsx125. doi:10.1093/sleep/zsx125

37. Jensen CD, Duraccio KM, Barnett KA, et al. Sleep duration differentially affects brain activation in response to food images in adolescents with overweight/obesity compared to adolescents with normal weight. Sleep. 2019;42(4):zsz001. doi:10.1093/sleep/zsz001

38. McGlinchey EL, Talbot LS, Chang KH, Kaplan KA, Dahl RE, Harvey AG. The effect of sleep deprivation on vocal expression of emotion in adolescents and adults. Sleep. 2011;34(9):1233-1241. doi:10.5665/SLEEP.1246

39. Talbot LS, McGlinchey EL, Kaplan KA, Dahl RE, Harvey AG. Sleep deprivation in adolescents and adults: changes in affect. Emotion. 2010;10(6):831-841. doi:10.1037/a0020138

40. Yoo SS, Gujar N, Hu P, Jolesz FA, Walker MP. The human emotional brain without sleep-a prefrontal amygdala disconnect. Curr Biol. 2007;17(20):R877-R878. doi:10.1016/j.cub.2007.09.008

41. Aparicio E, Canals J, Voltas N, Valenzano A, Arija V. Emotional symptoms and dietary patterns in early adolescence: a school-based follow-up study. J Nutr Educ Behav. 2017;49(5):405-414.e401. doi:10.1016/j.jneb.2017.01.015

42. Fulkerson JA, Sherwood NE, Perry CL, Neumark-Sztainer D, Story M. Depressive symptoms and adolescent eating and health behaviors: a multifaceted view in a population-based sample. Prev Med. 2004;38(6):865-875. doi:10.1016/j.ypmed.2003.12.028

43. Gibson EL. Emotional influences on food choice: sensory, physiological and psychological pathways. Physiol Behav. 2006;89(1):5361. doi:10.1016/j.physbeh.2006.01.024

44. Jaaskelainen A, Nevanpera N, Remes J, Rahkonen F, Jarvelin MR, Laitinen J. Stress-related eating, obesity and associated behavioural traits in adolescents: a prospective population-based cohort study. BMC Public Health. 2014;14:321. doi:10.1186/1471-2458-14-321

45. Farhangi MA. Night eating syndrome and its relationship with emotional eating, sleep quality and nutritional status among adolescents' boys. Community Ment Health J. 2019. doi:10.1007/ s10597-019-00395-8

46. Demirci E. Non suicidal self-injury, emotional eating and insomnia after child sexual abuse: are those symptoms related to emotion regulation? J Forensic Leg Med. 2018;53:17-21. doi:10.1016/j. jflm.2017.10.012

47. Ievers-Landis CE, Kneifel A, Giesel J, et al. Dietary intake and eating-related cognitions related to sleep among adolescents who are overweight or obese. J Pediatr Psychol. 2016;41(6):670-679. doi:10.1093/jpepsy/jsw017

48. Chardon ML, Janicke DM, Carmody JK, Dumont-Driscoll MC Youth internalizing symptoms, sleep-related problems, and disordered eating attitudes and behaviors: a moderated mediation analysis. Eat Behav. 2016;21:99-103. doi:10.1016/j.eatbeh.2016.01.007

49. Kelly NR, Shomaker LB, Radin RM, et al. Associations of sleep duration and quality with disinhibited eating behaviors in adolescent girls at-risk for type 2 diabetes. Eat Behav. 2016;22:149-155. doi:10.1016/j.eatbeh.2016.06.019

50. Klok MD, Jakobsdottir S, Drent ML. The role of leptin and ghrelin in the regulation of food intake and body weight in humans: a review. Obes Rev. 2007;8(1):21-34. doi:10.1111/j.1467-789X.2006.00270.x

51. Al-Disi D, Al-Daghri N, Khanam L, et al. Subjective sleep duration and quality influence diet composition and circulating adipocytokines and ghrelin levels in teen-age girls. Endocr J. 2010;57 (10):915-923.

52. Boeke CE, Storfer-Isser A, Redline S, Taveras EM. Childhood sleep duration and quality in relation to leptin concentration in two cohort studies. Sleep. 2014;37(3):613-620. doi:10.5665/sleep.3510 
53. Li L, Fu J, Yu XT, et al. Sleep duration and cardiometabolic risk among chinese school-aged children: do adipokines play a mediating role? Sleep. 2017;40(5):zsx042. doi:10.1093/sleep/zsx042

54. Kurtoglu S, Hatipoglu N, Mazicioglu M, Kendirici M, Keskin M, Kondolot M. Insulin resistance in obese children and adolescents: HOMA-IR cut-off levels in the prepubertal and pubertal periods. $J$ Clin Res Pediatr Endocrinol. 2010;2(3):100-106. doi:10.4274/jcrpe. v2i3.100

55. Templeman NM, Skovso S, Page MM, Lim GE, Johnson JD. A causal role for hyperinsulinemia in obesity. J Endocrinol. 2017;232 (3):R173-R183. doi:10.1530/JOE-16-0449

56. Moran A, Jacobs DR Jr, Steinberger J, et al. Insulin resistance during puberty: results from clamp studies in 357 children. Diabetes. 1999;48 (10):2039-2044. doi:10.2337/diabetes.48.10.2039

57. Kaar JL, Simon SL, Schmiege SJ, Nadeau KJ, Kelsey MM. Adolescent's health behaviors and risk for insulin resistance: a review of the literature. Curr Diab Rep. 2017;17(7):49. doi:10.1007/s11892-017-0902-3

58. De Bernardi Rodrigues AM, Da Silva Cde C, Vasques AC, et al. Association of sleep deprivation with reduction in insulin sensitivity as assessed by the hyperglycemic clamp technique in adolescents. JAMA Pediatr. 2016;170(5):487-494. doi:10.1001/jamapediatrics.2015.4365

59. Dorenbos E, Rijks JM, Adam TC, Westerterp-Plantenga MS, Vreugdenhil AC. Sleep efficiency as a determinant of insulin sensitivity in overweight and obese adolescents. Diabetes Obes Metab. 2015;17(Suppl 1):90-98. doi:10.1111/dom.12515

60. Klingenberg L, Chaput JP, Holmback U, et al. Acute sleep restriction reduces insulin sensitivity in adolescent boys. Sleep. 2013;36 (7):1085-1090. doi:10.5665/sleep.2816

61. Simon SL, Behn CD, Cree-Green M, et al. Too late and not enough: school year sleep duration, timing, and circadian misalignment are associated with reduced insulin sensitivity in adolescents with overweight/obesity. J Pediatr. 2019;205:257-264.e251. doi:10.1016/j. jpeds.2018.10.027

62. Javaheri S, Storfer-Isser A, Rosen CL, Redline S. Association of short and long sleep durations with insulin sensitivity in adolescents. $J$ Pediatr. 2011;158(4):617-623. doi:10.1016/j.jpeds.2010.09.080

63. Hagen EW, Starke SJ, Peppard PE. The association between sleep duration and leptin, ghrelin, and adiponectin among children and adolescents. Curr Sleep Medi Rep. 2015;1(4):185-194. doi:10.1007/ s40675-015-0025-9

64. Golley RK, Maher CA, Matricciani L, Olds TS. Sleep duration or bedtime? Exploring the association between sleep timing behaviour, diet and BMI in children and adolescents. Int $J$ Obes (Lond). 2013;37(4):546-551. doi:10.1038/ijo.2012.212

65. Beebe DW, Simon S, Summer S, Hemmer S, Strotman D, Dolan LM. Dietary intake following experimentally restricted sleep in adolescents. Sleep. 2013;36(6):827-834. doi:10.5665/ sleep. 2704

66. Beebe DW, Zhou A, Rausch J, Noe O, Simon SL. The impact of early bedtimes on adolescent caloric intake varies by chronotype. $J$ Adolesc Health. 2015;57(1):120-122. doi:10.1016/j.jadohealth.2015.02.017

67. Arora T, Taheri S. Associations among late chronotype, body mass index and dietary behaviors in young adolescents. Int $J$ Obes (Lond). 2015;39(1):39-44. doi:10.1038/ijo.2014.157

68. He F, Bixler EO, Berg A, et al. Habitual sleep variability, not sleep duration, is associated with caloric intake in adolescents. Sleep Med. 2015;16(7):856-861. doi:10.1016/j.sleep.2015.03.004

69. He F, Bixler EO, Liao J, et al. Habitual sleep variability, mediated by nutrition intake, is associated with abdominal obesity in adolescents. Sleep Med. 2015;16(12):1489-1494. doi:10.1016/j.sleep.2015.07.028

70. Sagala NJ, Sofyani S, Supriatmo S. Association between sleep quality and obesity in adolescents. Paediatr Indones. 2017;57 (1):41-46. doi:10.14238/pi57.1.2017
71. Al-Hazzaa HM, Musaiger AO, Abahussain NA, Al-Sobayel HI, Qahwaji DM. Lifestyle correlates of self-reported sleep duration among Saudi adolescents: a multicentre school-based cross-sectional study. Child Care Health Dev. 2014;40(4):533-542. doi:10.1111/ cch. 12051

72. Kruger AK, Reither EN, Peppard PE, Krueger PM, Hale L. Do sleep-deprived adolescents make less-healthy food choices? $\mathrm{Br} J$ Nutr. 2014;111(10):1898-1904. doi:10.1017/S0007114514000130

73. Ferranti R, Marventano S, Castellano S, et al. Sleep quality and duration is related with diet and obesity in young adolescent living in Sicily, Southern Italy. Sleep Sci. 2016;9(2):117-122. doi:10.1016/j.slsci.2016.04.003

74. Honkala S, Behbehani JM, Honkala E. Daily consumption of sugary drinks and foods as a behavioural risk for health of adolescents in Kuwait. Oral Health Prev Dent. 2012;10 (2):113-122.

75. Stea TH, Knutsen T, Torstveit MK. Association between short time in bed, health-risk behaviors and poor academic achievement among Norwegian adolescents. Sleep Med. 2014;15(6):666-671. doi:10.1016/j.sleep.2014.01.019

76. de Castro JM. When, how much and what foods are eaten are related to total daily food intake. Br J Nutr. 2009;102(8):12281237. doi:10.1017/S0007114509371640

77. Al-Haifi AA, AlMajed HT, Al-Hazzaa HM, Musaiger AO, Arab MA, Hasan RA. Relative contribution of obesity, sedentary behaviors and dietary habits to sleep duration among Kuwaiti adolescents. Glob J Health Sci. 2015;8(1):107-117. doi:10.5539/gjhs. v8n1p107

78. Gong QH, Li H, Zhang XH, Zhang T, Cui J, Xu GZ. Associations between sleep duration and physical activity and dietary behaviors in Chinese adolescents: results from the youth behavioral risk factor surveys of 2015. Sleep Med. 2017;37:168-173. doi:10.1016/j.sleep.2017.06.024

79. Tambalis KD, Panagiotakos DB, Psarra G, Sidossis LS. Insufficient sleep duration is associated with dietary habits, screen time, and obesity in children. J Clin Sleep Med. 2018;14(10):1689-1696. doi: $10.5664 /$ jcsm. 7374

80. Wang M, Zhong JM, Wang $\mathrm{H}$, et al. Breakfast consumption and its associations with health-related behaviors among school-aged adolescents: a cross-sectional study in Zhejiang Province, China. Int J Environ Res Public Health. 2016;13(8). doi:10.3390/ijerph13121252

81. Rossbach S, Diederichs T, Nothlings U, Buyken AE, Alexy U. Relevance of chronotype for eating patterns in adolescents. Chronobiol Int. 2018;35(3):336-347. doi:10.1080/07420528. 2017.1406493

82. Boschloo A, Ouwehand C, Dekker S, et al. The relation between breakfast skipping and school performance in adolescents. Mind Brain Educ. 2012;6(2):81-88. doi:10.1111/j.1751-228X.2012.01138.x

83. Otsuka Y, Kaneita Y, Itani O, et al. Association between unhealthy dietary behaviors and sleep disturbances among Japanese adolescents: a nationwide representative survey. Sleep Bio Rhythms. 2019;17(1):93-102. doi:10.1007/s41105-018-0193-3

84. Nasim M, Saade M, AlBuhairan F. Sleep deprivation: prevalence and associated factors among adolescents in Saudi Arabia. Sleep Med. 2019;53:165-171. doi:10.1016/j.sleep.2018.08.031

85. Cullen KW, Chen T-A. The contribution of the USDA school breakfast and lunch program meals to student daily dietary intake. Prev Med Rep. 2016;5:82-85. doi:10.1016/j. pmedr.2016.11.016

86. Collings PJ, Wijndaele K, Corder K, et al. Magnitude and determinants of change in objectively-measured physical activity, sedentary time and sleep duration from ages 15 to $17.5 \mathrm{y}$ in UK adolescents: the ROOTS study. Int J Behav Nutr Phys Act. 2015;12:61. doi:10.1186/s12966-015-0222-4 
87. Hayes JF, Balantekin KN, Altman M, Wilfley DE, Taylor CB, Williams J. Sleep patterns and quality are associated with severity of obesity and weight-related behaviors in adolescents with overweight and obesity. Child Obes. 2018;14(1):11-17. doi:10.1089/chi.2017.0148

88. Van Dyk TR, Krietsch KN, Saelens BE, Whitacre C, McAlister S, Beebe DW. Inducing more sleep on school nights reduces sedentary behavior without affecting physical activity in short-sleeping adolescents. Sleep Med. 2018;47:7-10. doi:10.1016/j.sleep.2018.03.007

89. Steele MM, Richardson B, Daratha KB, Bindler RC. Multiple behavioral factors related to weight status in a sample of early adolescents: relationships of sleep, screen time, and physical activity. Child Health Care. 2012;41(4):269-280. doi:10.1080/02739615.2012.721721

90. Lee J. Sleep duration's association with diet, physical activity, mental status, and weight among Korean high school students. Asia Pac J Clin Nutr. 2017;26(5):906-913. doi:10.6133/apjcn.082016.04

91. Bel S, Michels N, De Vriendt T, et al. Association between selfreported sleep duration and dietary quality in European adolescents. Br J Nutr. 2013;110(5):949-959. doi:10.1017/S0007114512006046

92. Hancox RJ, Poulton R. Watching television is associated with childhood obesity: but is it clinically important? Int $J$ Obes (Lond). 2006;30(1):171-175. doi:10.1038/sj.ijo.0803071

93. National Sleep Foundation. 2014 sleep in America poll - sleep in the modern family: summary of findings; 2014; Available from: http://sleepfoundation.org/sleep-polls-data/2014-sleep-the-modernfamily. Accessed July 10, 2015.

94. Carter B, Rees P, Hale L, Bhattacharjee D, Paradkar MS. Association between portable screen-based media device access or use and sleep outcomes: a systematic review and meta-analysis. JAMA Pediatr. 2016;170(12):1202-1208. doi:10.1001/jamapediatrics.2016.2341

95. Jun N, Lee A, Baik I. Associations of caffeinated beverage consumption and screen time with excessive daytime sleepiness in korean high school students. Clin Nutr Res. 2017;6(1):55-60. doi:10.7762/cnr.2017.6.1.55

96. Drescher AA, Goodwin JL, Silva GE, Quan SF. Caffeine and screen time in adolescence: associations with short sleep and obesity. $J$ Clin Sleep Med. 2011;7(4):337-342. doi:10.5664/JCSM.1182

97. Garaulet M, Ortega FB, Ruiz JR, et al. Short sleep duration is associated with increased obesity markers in European adolescents: effect of physical activity and dietary habits. The HELENA study. Int J Obes (Lond). 2011;35(10):1308-1317. doi:10.1038/ijo.2011.149

98. Paiva T, Gaspar T, Matos MG. Mutual relations between sleep deprivation, sleep stealers and risk behaviours in adolescents. Sleep Sci. 2016;9(1):7-13. doi:10.1016/j.slsci.2016.02.176

99. Gamble AL, D'Rozario AL, Bartlett DJ, et al. Adolescent sleep patterns and night-time technology use: results of the Australian broadcasting corporation's big sleep survey. PLoS One. 2014;9(11) e111700. doi:10.1371/journal.pone. 0111700

100. Polos PG, Bhat S, Gupta D, et al. The impact of Sleep Time-Related Information and Communication Technology (STRICT) on sleep patterns and daytime functioning in American adolescents. $J$ Adolesc. 2015;44:232-244. doi:10.1016/j.adolescence.2015.08.002

101. Children and parents: media use and attitudes report: 2013. Ofcom. Available from: https://www.ofcom.org.uk/research-and-data/ media-literacy-research/childrens/children-parents-oct-2013.

Published August 6, 2018. Accessed August 1, 2019.

102. Toh SH, Howie EK, Coenen P, Straker LM. "From the moment I wake up I will use it ... every day, very hour": a qualitative study on the patterns of adolescents' mobile touch screen device use from adolescent and parent perspectives. BMC Pediatr. 2019;19(1):30. doi:10.1186/s12887-019-1399-5
103. Laposky AD, Van Cauter E, Diez-Roux AV. Reducing health disparities: the role of sleep deficiency and sleep disorders. Sleep Med. 2016;18:3-6. doi:10.1016/j.sleep.2015.01.007

104. Guglielmo D, Gazmararian JA, Chung J, Rogers AE, Hale L. Racial/ethnic sleep disparities in US school-aged children and adolescents: a review of the literature. Sleep Health. 2018;4 (1):68-80. doi:10.1016/j.sleh.2017.09.005

105. Spruyt K, Alaribe CU, Nwabara OU. To sleep or not to sleep: a repeated daily challenge for African American children. CNS Neurosci Ther. 2015;21(1):23-31. doi:10.1111/cns.12319

106. El-Sheikh M, Bagley EJ, Keiley M, Elmore-Staton L, Chen E, Buckhalt JA. Economic adversity and children's sleep problems: multiple indicators and moderation of effects. Health Psychol. 2013;32(8):849-859. doi:10.1037/a0030413

107. Hale L, Berger LM, LeBourgeois MK, Brooks-Gunn J. Social and demographic predictors of preschoolers' bedtime routines. JDBP. 2009;30(5):394. doi:10.1097/DBP.0b013e3181b0f01b

108. Wilson KE, Miller AL, Bonuck K, Lumeng JC, Chervin RD. Evaluation of a sleep education program for low-income preschool children and their families. Sleep. 2014;37(6):1117-1125. doi:10.5665/sleep.3774

109. Keyes KM, Maslowsky J, Hamilton A, Schulenberg J. The great sleep recession: changes in sleep duration among US adolescents, 1991-2012. Pediatrics. 2015;135(3):460. doi:10.1542/peds.20141115

110. Saelens BE, Sallis JF, Frank LD, et al. Obesogenic neighborhood environments, child and parent obesity: the neighborhood impact on kids study. Am J Prev Med. 2012;42(5):e57-e64. doi:10.1016/j. amepre.2011.10.013

111. Rooney BL, Mathiason MA, Schauberger CW. Predictors of obesity in childhood, adolescence, and adulthood in a birth cohort. Matern Child Health J. 2011;15(8):1166-1175. doi:10.1007/ s10995-010-0689-1

112. Knutson KL. Sex differences in the association between sleep and body mass index in adolescents. $J$ Pediatr. 2005;147(6):830-834. doi:10.1016/j.jpeds.2005.07.019

113. Valrie CR, Bond K, Lutes LD, Carraway M, Collier DN. Relationship of sleep quality, baseline weight status, and weightloss responsiveness in obese adolescents in an immersion treatment program. Sleep Med. 2015;16(3):432-434. doi:10.1016/j. sleep.2014.11.007

114. Sallinen BJ, Hassan F, Olszewski A, et al. Longer weekly sleep duration predicts greater 3-month BMI reduction among obese adolescents attending a clinical multidisciplinary weight management program. Obes Facts. 2013;6(3):239-246. doi:10.1159/ 000351819

115. Tan E, Healey D, Gray AR, Galland BC. Sleep hygiene intervention for youth aged 10 to 18 years with problematic sleep: a beforeafter pilot study. BMC Pediatr. 2012;12:189. doi:10.1186/14712431-12-34

116. Minges KE, Redeker NS. Delayed school start times and adolescent sleep: a systematic review of the experimental evidence. Sleep Med Rev. 2016;28:86-95. doi:10.1016/j.smrv.2015.06.002

117. Gariepy G, Janssen I, Sentenac M, Elgar FJ. School start time and the healthy weight of adolescents. J Adolesc Health. 2018;63 (1):69-73. doi:10.1016/j.jadohealth.2018.01.009 


\section{Publish your work in this journal}

Adolescent Health, Medicine and Therapeutics is an international, peer-reviewed, open access journal focusing on health, pathology, and treatment issues specific to the adolescent age group. All aspects of health maintenance, preventative measures and disease treatment interventions are addressed within the journal and practitioners from

all disciplines are invited to submit their work as well as healthcare researchers and patient support groups. The manuscript management system is completely online and includes a very quick and fair peerreview system. Visit http://www.dovepress.com/testimonials.php to read real quotes from published authors. 